\title{
A Experiência do Usuário no desenvolvimento e avaliação de Softwares Educacionais
}

\author{
Mariana dos Santos Martins - PPGECMT - UDESC \\ mrs.mariana.martins@gmail.com \\ Avanilde Kemczinski - PPGECMT e PPGCA - UDESC \\ avanilde.kemczinski@udesc.br \\ Kariston Pereira - PPGECMT - UDESC \\ kariston.pereira@udesc.br \\ Isabela Gasparini - PPGECMT e PPGCA - UDESC \\ isabela.gasparini@udesc.br
}

Resumo. O conceito de experiência do usuário (UX) tem sido amplamente estudado e abordado no desenvolvimento de sistemas, a fim de tornar a ferramenta desenvolvida cada vez mais fácil e atraente para o usuário final. No entanto, ainda vemos pouca aplicabilidade deste conceito no campo de desenvolvimento de softwares educacionais (SE). O objetivo desta pesquisa é verificar como e em quais etapas a UX vem sendo utilizada dentro dos processos de desenvolvimento e avaliação de SEs por meio de um Mapeamento Sistemático da Literatura (MSL). Os dados coletados na pesquisa apontam que este conceito ainda é pouco utilizado nas etapas de desenvolvimento do produto educacional, sendo mais comumente visto nos processos de avaliação.

Palavras-chave: UX, software educacional, processo de desenvolvimento, avaliação de software.

\section{The user experience on the development and evaluation of Educational Software}

Abstract. The User eXperience (UX) concept has been widely studied and used in the systems development field, in order to make the use of the developed tool easier and more attractive for the final user. However, there is still few applicability of this concept in the field of educational development software. The goal of this research is to verify how and in which phases $U X$ has been applied in the development of the educational software using the Systematic Mapping of Literature method. The research points that UX is less used in the development phases of educational field, being more common in the evaluation processes.

Keywords: UX, Educational Software, Development Process, Software Evaluation.

\section{Introdução}

O termo experiência do usuário (User Experience - UX) foi popularizado durante a década de 1990 e, desde então, a $U X$ tem sido amplamente estudada e abordada no desenvolvimento de sistemas em geral. A $U X$ envolve o modo como o uso de sistemas interativos afetam os sentimentos e as emoções do usuário (Barbosa e Silva 2010). UX é o conjunto de elementos e fatores relativos à interação do usuário com um determinado produto, sistema ou serviço cujo resultado gera uma percepção positiva ou negativa. $O$ termo está relacionado a aspectos mais subjetivos, pois trata da percepção e comportamento do usuário frente a um sistema, produto ou serviço.

Este trabalho tem como objetivo verificar como a $U X$ está sendo utilizada em Softwares Educacionais (SEs). Se faz necessário mapear quais métodos e técnicas estão sendo utilizados para alcançar aspectos positivos da $U X$ e em que fase do desenvolvimento do software eles ocorrem (desenvolvimento ou avaliação), além disso busca-se elencar quais critérios de avaliação são utilizados dentro da avaliação do SE, e em que tipo de SE é mais comum observar aspectos de $U X$. 
Busca-se explorar neste artigo os aspectos da UX dentro do cenário de Softwares Educacionais (SE). Para tal a técnica de Mapeamento Sistemático da Literatura (MSL) foi utilizada. Para tanto, o artigo está dividido em Fundamentação Teórica (seção 2), Metodologia (seção 3), Resultados (seção 4) e as Considerações Finais (seção 5), seguidas das referências bibliográficas.

\title{
2. Experiência do Usuário
}

A experiência do usuário (do inglês User Experience - UX) tem grande potencial no momento de desenvolvimento de um software, seja para realizar a avaliação do produto ou para aperfeiçoar o seu design. "Mais especificamente, a experiência de usuário diz respeito a como as pessoas se sentem em relação a um produto e ao prazer e à satisfação que obtém ao usá-lo, olhá-lo, abrí-lo ou fechá-lo." (ROGERS, SHARP, PREECE, 2013, p. 13). Tullis e Albert (2008) definem três principais características para conceituar UX, são elas (1) o envolvimento do usuário; (2) a interação deste usuário com o produto, sistema, etc.; (3) se a experiência deste usuário é interessante, observável e mensurável. Sendo assim, para que haja UX é necessário que haja interação, comportamento, um produto e/ou uma interface.

\begin{abstract}
Apesar de a complexidade e a noção ainda não terem sido exploradas completamente, a experiência do usuário pode ser descrita como o conjunto de todos os processos (físicos, cognitivos, emocionais) desencadeados no usuário a partir da sua interação com um produto ou serviço em diversos momentos (que incluem a expectativa da interação, a interação propriamente dita e a reflexão após a interação) em um determinado contexto de uso (físico, social, tecnológico). (CYBIS; BETIOL; FAUST, 2015, p. 438).
\end{abstract}

Westerink et.al. (2008) afirmam que a experiência do usuário é um diferencial para garantir o sucesso do sistema ou produto, partindo apenas da avaliação da perspectiva do usuário através da interface do produto, será essa a "interação" a ser observada e medida, a partir das métricas de UX. "Ela abrange diferentes aspectos da experiência do usuário, desde como nos sentimos quando descobrimos pela primeira vez um novo produto até quando nos livramos dele." (ROGERS, SHARP, PREECE, 2013, p. 128). A UX tem o intuito de observar a interação total do indivíduo com o sistema ou componente de software, levando em consideração pensamentos, sentimentos e outras percepções que advêm dessa interação. Este conceito não está relacionado apenas a sistemas, segundo Tullis e Albert (2008) a UX também está relacionada a produtos físicos, e a maneira como os usuários fazem uso destes produtos "A experiência do usuário [...] resultam de expectativas, do uso efetivo e das reflexões sobre o uso do produto em um contexto individual e social." (CYBIS, BETIOL, FAUST, 2015, p. 192).

\subsection{A Usabilidade}

Usabilidade e experiência do usuário (UX) são dois critérios de qualidade de uso de sistemas que estão relacionados. Alguns autores trazem o termo de usabilidade para explicar a experiência do usuário, outros autores tratam os termos como sinônimos mas aqui iremos apresentar as diferenças entre estes dois critérios.

Segundo a norma ISO 9241-11 (1998) a usabilidade é o grau em que um produto é usado por usuários específicos para atingir objetivos específicos com eficácia, eficiência e satisfação em um contexto de uso específico. A usabilidade se distancia da experiência do usuário a medida que o aspecto mais subjetivo está relacionadoa satisfação em relação ao uso do produto, e os outros aspectos são mais quantitativos. " $\mathrm{O}$ que se pode depreender dos princípios de usabilidade é que eles tratam basicamente de dois aspectos: a tarefa e as características individuais dos usuários." (ROCHA, 2003, p. 
32). A usabilidade verifica a execução da tarefa, ela se pergunta "isso está funcional?", se preocupa se o usuário conseguirá localizar o próximo passo de uma sequência, finalizar uma tarefa dentro do sistema. "Esses critérios são geralmente explorados por perguntas específicas associadas a algum dado mensurável, que com frequência pode ser objetivamente capturado durante a interação do usuário com o sistema." (BARBOSA e SILVA, 2010, p. 341). "[...] [usabilidade] é a questão de saber se o sistema é suficientemente bom para satisfazer todas as necessidades e requisitos dos usuários e de outras partes interessadas em potencial, como os clientes e gerentes dos usuários." ${ }^{1}$ (NIELSEN, 1993, p. 24). "A usabilidade endereça principalmente a capacidade cognitiva, perceptiva e motora dos usuários empregada durante a interação." (BARBOSA, 2010, p. 29).

Já a UX trata da subjetividade dos usuários, e verifica se o produto é agradável e se o usuário gosta de realizar as atividades dentro do respectivo produto. O conceito de UX está mais atrelado ao marketing, busca um sentimento/sensação, antes mesmo do primeiro contato do produto, e também se preocupa com a "impressão" que o usuário levará do software.

\title{
2.2. Avaliação da Usabilidade e da Experiência do Usuário
}

A Associação dos Profissionais de Experiência do Usuário (UXPA) define a UX como uma abordagem ao desenvolvimento de produtos ou serviços que incorporam, além da avaliação, o feedback direto dos usuários durante o ciclo de desenvolvimento (Ramos et al., 2016). Goodman et.al. (2012) afirmam que no período de desenvolvimento (tanto nas fases iniciais, quanto nas intermediárias) o ideal é fazer avaliações em todas as etapas de desenvolvimento do produto.

A observação dos aspectos de UX exige mais do que o emprego de questionários para verificar os sentimentos e as emoções dos usuários, é necessário que haja um ambiente próprio para que possam ser coletados dados significativos quanto as reações do usuário em relação ao produto. Desta forma uma das técnicas a ser aplicada pode ser um Teste de Usabilidade.

Rubin e Chisnell (2008, p. 21) definem teste de usabilidade como uma ferramenta de pesquisa, com suas raízes na metodologia experimental clássica. A gama de testes que podemos realizar é considerável, desde experimentos clássicos com grandes tamanhos amostrais e desenhos de testes complexos até estudos qualitativos mais informais com poucos participantes.

\begin{abstract}
A usabilidade é tipicamente medida por um número de usuários de teste [...] usando o sistema para executar um conjunto de tarefas previamente especificados, embora também possa ser medido com usuários reais em campo realizando quaisquer tarefas que estejam fazendo de qualquer maneira (NIELSEN, 1993, p. 27). ${ }^{2}$
\end{abstract}

Goodman et.al. (2012) afirmam que testes de usabilidade são a porta para compreender como o usuário interpreta e usa o software, e são eles que podem dizer a diferença entre um produto que será útil e utilizável, de um fracasso dentro do mercado.

\footnotetext{
${ }^{1}[\ldots]$ (Usability) is the question of whether the system is good enough to satisfy all the needs and requirements of the users and other potential stakeholders, such as the users' clients and managers.

${ }^{2}$ Usability is typically measured by having a number of test users [...] use the system to perform a prespecified set of tasks, though it can also be measured by having real users in the field perform whatever tasks they are doing anyway.
} 


\section{Metodologia}

A metodologia utilizada para a realização desta pesquisa foi um Mapeamento Sistemático da Literatura, seguindo os passos e definições de Petersen et.al. (2008). De acordo com Petersen et.al. (2008) é necessária a realização dos seguintes passos: (1) Definição de questões de pesquisa; (2) Condução da busca (String de pesquisa); (3) Seleção dos trabalhos (Critérios de Inclusão e Exclusão); (4) Análise e Mapeamento dos Dados obtidos. Todos os passos serão desenvolvidos nos tópicos seguintes.

\subsection{Questões de Pesquisa}

A questão de pesquisa que norteia o andamento desta perquisa é "Como a UX é utilizada/aplicada em softwares educativos?" (Q1). Outras perguntas foram desenvolvidas para auxiliar na resolução do problema principal de pesquisa:

- Como a UX é aplicada no decorrer do desenvolvimento do SE?

- Como a UX acontece na avaliação final do SE?

- Quais os tipos de SEs que utilizam a metodologia UX (tanto em seu desenvolvimento quanto em sua avaliação final)?

\subsection{Condução da Busca}

Para a realização da pesquisa foram utilizados quatro Mecanismos de Busca Acadêmica (MBA), foram eles: IEEE Explorer, Google Scholar, Springer Link e Engineering Village. Os mecanismos foram escolhidos com base nos critérios aplicados por Buchinger et.al. (2014), na avaliação quantitativa de 40 MBAs.

A string de pesquisa foi definida após uma rodada de testes, no entanto, ela foi deixada em seu modo mais amplo, devido à escassez de trabalhos que se apresentaram nessa área. A string definida em português foi: ("UX" OR "Experiência do Usuário") AND ("Software Educativo" OR "Software Educacional") AND ("Avaliação de Software"). Enquanto a string definida em inglês foi definida como: ("UX" OR "User Experience") AND ("Educational Software") AND ("Software Evaluation").

Os artigos selecionados por meio da string de busca no MBA analisaram os títulos, o resumo e as palavras-chave. Foram considerados artigos entre os anos de 2013 à 2018 .

\subsection{Seleção dos Trabalhos}

A seleção dos trabalhos a serem lidos se dá por meio do emprego de critérios de inclusão e exclusão, estes são aplicados a todas as obras que aparecem após a aplicação da string de busca. Como critérios de inclusão (CIs) têm-se:

- Artigos disponíveis para download (arquivos de acesso irrestrito e gratuitos);

- Artigos publicados em revistas científicas ou conferências (levando em consideração o rigor científico desses meios de publicação);

- Artigos escritos em inglês ou português (língua de domínio dos autores);

- Artigos que tratem da UX em Softwares Educacionais (escopo do trabalho). Como critérios de exclusão (CEs) foram definidos:

- Artigos duplicados;

- Artigos secundários (MSLs e Revisões Sistemáticas da Literatura - RSLs);

- Artigos anteriores ao ano de 2013.

O corte realizado pela data como $\mathrm{CE}$ se deu para levar em consideração a atualidade dos trabalhos desenvolvidos, apesar o termo UX ter se popularizado nos anos 90, ainda hoje não se vê grande aplicação do mesmo no desenvolvimento de artefatos tecnológicos educacionais.

Com a aplicação da string, foram encontrados ao todo 234 artigos relacionados. 
Após a aplicação dos critérios de inclusão e exclusão, restaram 12 artigos. Os resultados podem ser mais bem visualizados na tabela 1 .

Tabela 1 - Resultados da pesquisa de acordo com os MBAs utilizados

\begin{tabular}{|c|c|c|c|}
\hline MBAs & $\begin{array}{c}\text { Resultado da } \\
\text { string }\end{array}$ & CIs & CEs \\
\hline $\begin{array}{c}\text { Engineering } \\
\text { Village }\end{array}$ & 33 & 6 & 2 \\
\hline Google Scholar & 68 & 32 & 7 \\
\hline IEEE & 126 & 6 & 2 \\
\hline Springer Link & 270 & 26 & 1 \\
\hline Total & 234 & 70 & 12 \\
\hline
\end{tabular}

\subsection{Análise e Mapeamentos dos Dados}

Todos os artigos selecionados após a utilização dos CIs e CEs foram lidos integralmente. As informações retiradas dos artigos fazem referência direta às questões de pesquisa apresentadas anteriormente. Os dados extraídos foram: tipo de SE avaliado; momento de aplicação do UX (durante o desenvolvimento do produto ou como avaliação final); método de aplicação do UX/teste de usabilidade.

\section{Resultados}

Levando em consideração as questões de pesquisa, durante o levantamento de dados surgiram outras curiosidades a respeito do uso do UX em SEs. Dos 12 artigos estudados, apenas 3 trouxeram o termo user experience, se referindo à UX como critério de qualidade de uso do sistema, e não à experiência do usuário como termo para descrever o que é medido por meio de um teste de usabilidade.

Dentre os 12 artigos, 9 deles utilizaram UX, ou algum teste de usabilidade somente na avaliação final do produto, enquanto 3 deles fizeram o uso destes conceitos no decorrer do desenvolvimento do SE. Os resultados podem ser mais bem visualizados na Figura 1.

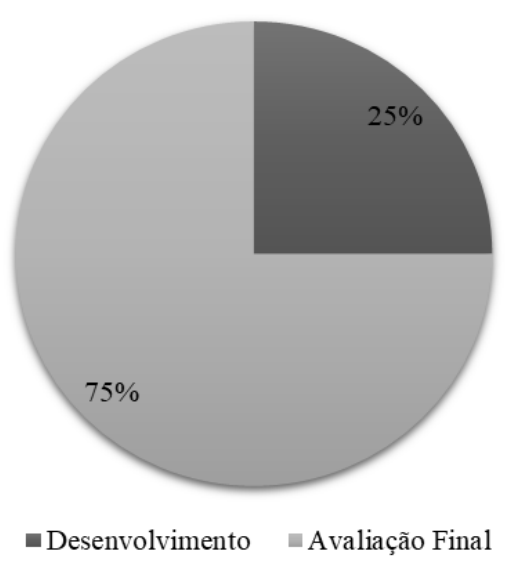

Figura 1 - Momento de utilização da UX/teste de usabilidade

As técnicas de Testes de Usabilidade ou UX são descritas nas Tabelas 2 e 3, sendo separadas pelo momento da aplicação da metodologia, podendo ser utilizada no desenvolvimento do produto, ou em sua avaliação final. Como consequência desta pesquisa pôde-se observar diferentes técnicas, desde questionários elaborados pelos próprios pesquisadores, ou questionários da literatura, como o SUS (System Usability Scale). Para a descrição nas tabelas, respeitou-se a nomenclatura dada pelos próprios 
autores de cada trabalho, para definir a metodologia, produto e ferramentas utilizadas durante a avaliação.

Tabela 2 - Técnicas de avaliação durante desenvolvimento do produto

\begin{tabular}{|c|c|c|c|c|c|}
\hline Autores & Metodologia Utilizada & Produto & \multicolumn{2}{c|}{ Ferramentas de Avaliação } & Total \\
\hline \multicolumn{5}{|c|}{ Avaliação durante o Desenvolvimento do Produto } \\
\hline $\begin{array}{c}\text { Neves e Kanda } \\
\text { (2016). }\end{array}$ & Teste de usabilidade & Jogo & $\begin{array}{l}\text { Questionário desenvolvido } \\
\text { pelos pesquisadores } \\
\text { Observação da utilização }\end{array}$ & 2 \\
\hline Sun (2017). & Teste de usabilidade & LMS & $\begin{array}{l}\text { Algoritmo desenvolvido } \\
\text { pelos pesquisadores } \\
\text { Avaliação por } \\
\text { especialistas }\end{array}$ & 2 \\
\hline $\begin{array}{c}\text { Landowska e } \\
\text { Miler (2016). }\end{array}$ & UX & Jogo & Questionário desenvolvido & pelos pesquisadores \\
Questionário SUS & 2 \\
\hline
\end{tabular}

Tabela 3 - Técnicas de avaliação final do produto

\begin{tabular}{|c|c|c|c|c|}
\hline Autores & Metodologia Utilizada & Produto & Ferramentas de Avaliação & Total \\
\hline \multicolumn{5}{|c|}{ Avaliação final do Produto } \\
\hline $\begin{array}{l}\text { Santos, et.al. } \\
\text { (2017). }\end{array}$ & UX & ARLE & 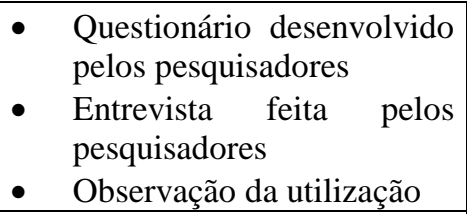 & 3 \\
\hline $\begin{array}{l}\text { Flores e Gamboa } \\
\qquad(2015) .\end{array}$ & Teste de Usabilidade & LMS & $\begin{array}{l}\text { - Questionário desenvolvido } \\
\text { pelos pesquisadores } \\
\text { - } \quad \text { Avaliação por especialistas }\end{array}$ & 2 \\
\hline $\begin{array}{l}\text { Nascimente, } \\
\text { et.al. }(2016) \text {. }\end{array}$ & UX & APP & $\begin{array}{ll}\text { - } & \text { Questionário Userbility } \\
\text { - } & \text { Questionário UUGA }\end{array}$ & 2 \\
\hline $\begin{array}{l}\text { Falcão, et.al. } \\
\qquad(2015) .\end{array}$ & Teste de usabilidade & Jogo & $\begin{array}{ll}\text { - } & \text { Observação da utilização } \\
\text { - } & \text { Anotações }\end{array}$ & 2 \\
\hline $\begin{array}{l}\text { Papachristos, } \\
\text { et.al. }(2013) \text {. }\end{array}$ & Teste de Usabilidade & Simulador & $\begin{array}{l}\text { - } \begin{array}{l}\text { Questionário desenvolvido } \\
\text { pelos pesquisadores } \\
\text { - Entrevista feita pelos } \\
\text { pesquisadores }\end{array} \\
\end{array}$ & 2 \\
\hline $\begin{array}{l}\text { Herodotou, et.al. } \\
\qquad(2018) .\end{array}$ & Teste de usabilidade & APP & $\begin{array}{ll}\text { - } & \text { Observação da utilização } \\
\text { - } & \text { Anotações }\end{array}$ & 2 \\
\hline $\begin{array}{l}\text { Orfanou, et.al. } \\
\quad(2015) \text {. }\end{array}$ & Teste de usabilidade & LMS & - Questionário SUS & 1 \\
\hline $\begin{array}{c}\text { Althobaiti1 e } \\
\text { Mayhew (2016). }\end{array}$ & Teste de Usabilidade & LMS & $\begin{array}{l}\text { - Questionário desenvolvido } \\
\text { pelos pesquisadores }\end{array}$ & 1 \\
\hline $\begin{array}{l}\text { Marciano, et.al. } \\
\text { (2014). }\end{array}$ & Teste de Usabilidade & Jogo & $\begin{array}{l}\text { - Questionário desenvolvido } \\
\text { pelos pesquisadores }\end{array}$ & 1 \\
\hline
\end{tabular}

Além disso, também pôde-se perceber a falta de metodologia para utilização e elaboração de Questionários para a realização de Testes de Usabilidade, como pode-se observar na Tabela 4, 7 dos 12 artigos apresentaram questionário elaborados pelos pesquisadores.

Tabela 4 - Verificação de utilização por ferramenta de avaliação

\begin{tabular}{|c|c|c|}
\hline \multicolumn{2}{|c|}{ Total } \\
\hline Ferramenta de Avaliação 1 & Questionário desenvolvido pelos pesquisadores & 7 \\
\hline Ferramenta de Avaliação 2 & Observação da utilização & 4 \\
\hline
\end{tabular}




\begin{tabular}{|l|c|c|}
\hline Ferramenta de Avaliação 3 & Anotações & 2 \\
\hline Ferramenta de Avaliação 4 & Avaliação por especialistas & 2 \\
\hline Ferramenta de Avaliação 5 & Entrevista feita pelos pesquisadores & 2 \\
\hline Ferramenta de Avaliação 6 & Questionário SUS & 2 \\
\hline Ferramenta de Avaliação 7 & Algorítmo desenvolvido pelos pesquisadores & 1 \\
\hline Ferramenta de Avaliação 8 & Questionário Userbility & 1 \\
\hline Ferramenta de Avaliação 9 & Questionário UX e Abordagem de Diretrizes de & 1 \\
\hline
\end{tabular}

Na pesquisa, se apresentaram diferentes tipos de SEs, entre eles jogos, Sistemas de Gerenciamento de Aprendizagem (LMS) e, ainda, um tipo de software que não era esperado nos resultados, o software de aprendizagem que se utiliza de experimentos de realidade aumentada (ARLE). Na Figura 2 há a representação da quantidade e dos diferentes tipos de SEs que resultaram da pesquisa.

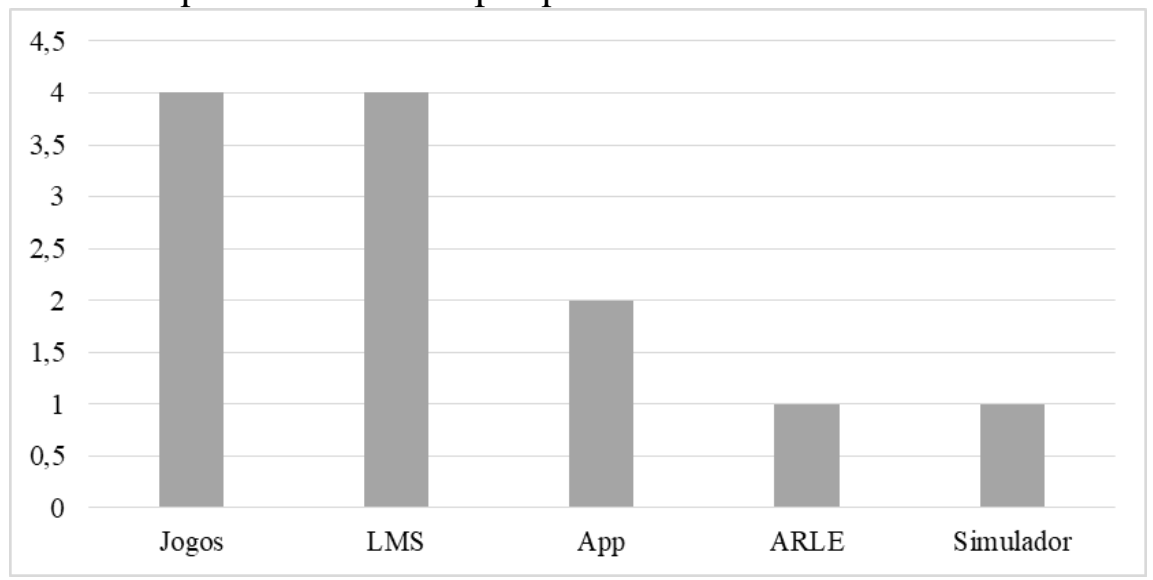

Figura 2 - Tipos de SEs

\section{Discussão}

Inicialmente, buscou-se identificar como a UX é utilizada nos SEs, mas pôde ser observado que somente 3 dos 12 trabalhos traziam o termo user experience como conceito por trás da avaliação. Portanto foram também levados em consideração trabalhos que realizavam algum tipo de Teste de Usabilidade, podendo ser utilizado durante o desenvolvimento ou no final do produto, para que, então, se pudesse sanar a Q1 da pesquisa.

Os resultados da pesquisa apontaram, na Figura 1, que em somente $25 \%$ dos casos o conceito de UX e Testes de Usabilidade são utilizados durante o desenvolvimento do SE, enquanto nos outros $75 \%$ eles são utilizados somente como avaliação final do produto. Lembrando que autores como Cybis, Betiol e Faust, (2015) reforçam a necessidade de avaliação durante o processo de desenvolvimento do produto, principalmente se trantando do conceito de UX.

Os autores Neves e Kanda (2016) afirmam em sua pesquisa que as avaliações realizadas durante o desenvolvimento do produto foram de extrema importância, pois por meio delas pôde-se notar caracteríticas importantes para que houvesse a permanente atenção dos alunos durante a utilização do SE (na pesquisa dos autores foi elaborado um protótipo do SE e, então, aplicado e avaliado, somente depois foi desenvolvido o jogo em si com as características observadas na avaliação de usabilidade do protótipo).

Os resultados da pesquisa apontam na Tabela 2, que durante o desenvolvimento do produto é mais comum vermos a utilização de Testes de Usabilidade (2/3 dos resultados da pesquisa utilizaram essa metodologia), mas esse volume também pode ser percebido na avaliação final, na Tabela 3 , onde $77 \%$ das pesquisas também utilizam 
Testes de Usabilidade. De modo geral, a Ferramenta de avaliação mais utilizada, tanto no desenvolvimento quanto no final do produto, foram os questionários desenvolvidos pelos próprios pesquisadores, esta ferramenta representa um total de $58 \%$ dentro da pesquisa. Esses resultados também nos fazem questionar a qualidade de avaliação do produto, como já vimos anteriormente, para que haja avaliação partindo do conceito de UX é necessário que haja um ambiente controlado (preferencialmente um laboratório), e isso não foi observado em nenhum dos estudos encontrados.

Os tipos de SEs encontrados na execução da pesquisa apontam, na Figura 2, que é mais comum que haja avaliação da experiência do usuário em jogos educacionais e em LMSs, compreendendo um total de $33 \%$ cada um; juntos compreendem mais da metade dos resultados obtidos.

De modo geral pode-se dizer que a avaliação de SEs com o uso da metodologia UX ainda é escassa no campo educacional. No entanto, Santos et.al. (2014) afirmam em sua pesquisa que medir a experiência do usuário é uma oportunidade de descobrir possíveis melhorias no produto, tornando-se, assim, imprescindível a utilização de tais metodologias na busca da excelência no desenvolvimento dos softwares educacionais.

\section{Considerações Finais}

O Mapeamento Sistemático da Literatura permitiu alcançar resultados positivos na coleta de dados realizada nesta pesquisa. A metodologia proporcionou além de vigor científico, auxilio na busca e delimitação das questões de pesquisa, mantendo-a focada em seus objetivos pré-definidos.

Com a observação dos resultados apresentados pela pesquisa pôde-se observar pouca aplicação da Metodologia UX/Testes de Usabilidade durante o desenvolvimento de softwares educacionais, sendo que eles foram mais comumente observados na avaliação final do produto, que representam $75 \%$ dentro da pesquisa, como observado no Figura 1. De maneira geral, a ferramenta mais utilizada para que haja a avaliação, tanto do desenvolvimento quanto no final do produto, é o questionário elaborado pelos próprios pesquisadores, demonstrando necessidade de amadurecimento de ferramentas avaliativas de usabilidade na área de avaliação de SEs. Entre os produtos mais avaliados temos os Jogos Educacionais e os LMSs.

É importante levar em consideração a experiência do usuário além da avaliação, trazendo-a para o desenvolvimento do software, para que ela possa ser mais bem utilizada no decorrer do desenvolvimento do produto, tornando a ferramenta criada mais atrativa, agradável e fácil ao seu usuário final.

A pesquisa aponta para uma utilização equivocada de Testes de Usabilidade como ferramentas para avaliação de UX. Se faz necessário, ainda, pesquisa mais aprofundada na heurística da utilização de UX em SEs, para que se possa entender por que a metodologia ainda é pouco utilizada na avaliação destes produtos. Como sugestão para trabalhos futuros deve-se considerar uma pesquisa afim de elencar as principais dificuldades encontradas para a aplicação do conceito de UX na avaliação de SEs, ou até mesmo na proposição de uma ferramenta de avaliação UX para validação e/ou elaboração de artefatos tecnológicos educacionais.

\section{Referências}

ALTHOBAITI, M.M.; MAYHEW, P. How usable are the learning management systems? the users have their say. In: EAI Endorsed Transactions on e-Learning. vol. $3,2016$. 
BARBOSA, Simone Diniz Junqueira; SILVA, Bruno Santana da. Interação humanocomputador. Rio de Janeiro: Elsevier, 2010.

CYBIS, Walter; BETIOL, Adriana Holtz; FAUST, Richard. Ergonomia e usabilidade: conhecimentos, métodos e aplicações. São Paulo: Novatec, 2015.

ELLWANGER, Cristiane; SANTOS, Cristina Paludo; LEVANDOWSKI, Joel. Aplicação do método AHP para avaliação da usabilidade de sistemas. In: HFD (Human Factors in Design), p. 5 - 16, 2013.

FALCÃO, Taciana Pontual; GOMES, Tancicleide C. Simões; ALBUQUERUE, Isabella Rocha. O pensamento computacional através de jogos infantis: uma análise de elementos de interação. In: Simpósio Brasileiro sobre Fatores Humanos em Sistemas Computacionais. 2015.

FLORES, Ruth Medina; GAMBOA, Rafael Morales. Usability evaluation by experts of a learning management system. In: IEEE Revista Iberoamericana de Tecnologias del Aprendizaje, vol. 10, p. 197 - 203, 2015.

GOODMAN, Elizabeth; KUNIAVSKY, Mike; MOED, Andrea. Observing the user experience: a practitioner's guide to user research. 2. ed. Massachusetts: Elsevier, 2012.

HERODOTOU, Christothea; ARISTEIDOU, Maria; SHARPLES, Mike; SCANLON, Eileen. Designing citizen science tools for learning: lessons learnt from the iterative development of nQuire. In: Research and Practice in Technology Enhanced Learning, 2018.

LANDOWSKA, Agnieszka; MILER, Jakub. Limitations of emotion recognition in software user experience: evaluation context. In: Proceedings of the Federated Conference on Computer Science and Information Systems, p. 1631-1640, 2016.

MARCIANO, Juvane Nunes; MIRANDA, Leonardo Cunha; MIRANDA, Erica Esteves Cunha de. Evaluating multiple aspects of educational computer games: literature review and case study. In: International Journal of Computer Games Technology. 12 p., 2014.

NASCIMENTO, Ingrid; GASPAR, Wagner; CONTE, Tayana; GADELHA, Bruno; OLIVEIRA, Elaine H. T. Melhor prevenir do que remediar: avaliando usabilidade e UX de software antes de levá-lo para a sala de aula. In: V Congresso Brasileiro de Informática na Educação (CBIE), p. 806 - 825, 2016.

NEVES, Libni Almeida; KANDA, Jorge Yoshio. Desenvolvimento e avaliação de jogos educativos para deficientes intelectuais. In: Nuevas Ideas en Informática Educativa. Vol. 12, p. $612-617,2016$.

NIELSEN, Jakob. Usability engineering. California: Morgan Kaufmann, 1993.

ORFANOU, Konstantina; TSELIOS, Nikolaos; KATSANOS, Christos. Perceived usability evaluation of learning management systems: empirical evaluation of the system usability scale. In: International Review of Research in Open and Distributed 
Learning. Volume 16, p. $227-246,2015$.

PAPACHRISTOS, D.; ALAFODIMOS, K.; KIKILIA, K.; NIKITAKOS, N. Sentiment analysis use in the satisfaction evaluation for maritime education. In: The International Conference on E-Learning in the Workplace. 2013.

PETERSEN, K.; FELDT, R.; MUJTABA, S.; MATTSSON, M. Systematic mapping studies in software engineering. In: Proceedings of the 12th international conference on Evaluation and Assessment in Software Engineering. P. 68-77, 2008.

RAMOS, Mayara; MERINO, Eugenio A. D.; MERINO, Giselle S. A. D.; FERREIRA, Marcelo G. G. Design de serviços e experiência do usuário (UX): uma análise do relacionamento das áreas. In: DAPesquisa, v.11, p. 105 - 1023, 2016.

RUBIN, Jeffrey; CHISNELL, Dana. Handbook of usability testing: how to plan, design, and conduct effective teste. 2. ed. Indianapolis: Wiley Publishing, 2008.

ROCHA, Heloisa Vieira da; BARANAUSKAS, Maria Cecília Calani. Design e avaliação de interfaces humano-computador. São Paulo: UNICAMP, 2003.

ROGERS, Yvonne; SHARP, Helen; PREECE, Jennifer. Design de interação: além da interação humano-computador. São Paulo: Bookman, 2013.

SANTOS, Marc Ericson C.; CHEN, Angie; TAKETOMI, Takafumi; YAMAMOTO, Goshiro; MIYAZAKI, Jun; KATO, Hirokazu. Augmented reality learning experiences: survey of prototype design and evaluation. In: IEEE Transactions On Learning Technologies, vol. 7, p. $38-56,2017$.

SUN, Jiaze. Usability Evaluation Approach of Educational Resources Software Using Mixed Intelligent Optimization. In: Mathematical Problems in Engineering, 2017.

TULLIS, Tom; ALBERT, Bill. Measuring the User Experience: collecting, analyzing, and presenting usability metrics. 2. ed. Massachusetts: Elsevier, 2008.

WESTERINK, Joyce H.; OUWERKERK, M.; OVERBEEK, T.; PASVEER, W Probing experience from assessment of user emotions and behaviour to development of products. Netherlands: Springer, 2008. 